\section{Expectativas positivas com o uso de álcool e o beber se embriagando: diferenças de gênero em estudo do Projeto GENACIS, São Paulo, Brasil}

\author{
Positive expectations towards alcohol use and binge \\ drinking: gender differences in a study from the \\ GENACIS project, São Paulo, Brazil
}

\author{
${ }^{1}$ Fundação de Ação Social, \\ Prefeitura Municipal de \\ Curitiba, Curitiba, Brasil. \\ 2 Faculdade de Filosofia \\ e Ciências, Universidade \\ Estadual Paulista Júlio de \\ Mesquita Filho, Marília, \\ Brasil. \\ 3 Faculdade de Medicina \\ de Botucatu, Universidade \\ Estadual Paulista Júlio de \\ Mesquita Filho, Botucatu, \\ Brasil. \\ Correspondência \\ M. C. P. Lima \\ Departamento de Neurologia \\ e Psiquiatria, Faculdade \\ de Medicina de Botucatu, \\ Universidade Estadual \\ Paulista Júlio de Mesquita \\ Filho. \\ Distrito de Rubião Jr. \\ Botucatu, SP \\ 18618-000, Brasil. \\ kika.botucatu@gmail.com
}

\begin{abstract}
The objective was to investigate expectations towards alcohol use among men and women and the association between these expectations and binge drinking (or heavy episodic drinking). An epidemiological cross-sectional populationbased household survey with a stratified probabilistic sample was conducted in Greater Metropolitan São Paulo, Brazil. Males and females were interviewed ( $n=2,083)$ with the GENACIS questionnaire (Gender, Alcohol, and Culture: an International Study). The dependent variable was binge drinking, and logistic regression models were constructed for each gender, adjusting for age, schooling, and income. All expectations, with the exception of increased ease in talking with one's partner, were associated with binge drinking. The study shows that binge drinking can be associated with expectations towards alcohol use. Understanding such expectations can contribute to the design of effective polices to prevent binge drinking.
\end{abstract}

Alcohol Drinking; Alcoholism; Behavior
Mariana Braga Cavariani 1 Janaina Barbosa de Oliveira 2 Florence Kerr-Corrêa ${ }^{3}$ Maria Cristina Pereira Lima ${ }^{3}$

\section{Introdução}

"Macduff: Quais as três coisas que o beber especificamente provoca?

Porter: ...nariz vermelho, sono e urina. Luxúria, senhor, ele provoca e não provoca; provoca o desejo, mas impede o desempenho" (William Shakespeare, Macbeth; act II, scene I).

No mundo todo, estima-se que cerca de 2 bilhões de pessoas façam uso de bebidas alcoólicas, e, dessas, 7 milhões apresentam algum distúrbio ou consequência danosa decorrente 1 . No Brasil, dados de um levantamento nacional demonstram que grande parte da população é abstinente (35\% entre os homens e $59 \%$ entre as mulheres); no entanto, aqueles que fazem uso de bebidas alcoólicas ingerem grandes quantidades. Segundo este estudo, 29\% dos entrevistados relataram, ao menos, um episódio de beber com embriaguez no ano anterior 2. Esse padrão, que consiste na ingestão de cinco ou mais drinques por ocasião, é considerado extremamente nocivo por estar associado a doenças como câncer, cirrose, transtornos mentais e também a acidentes e consequências sociais como homicídios, assaltos, assassinatos 3 e violência entre parceiros íntimos 4 . Ainda no inquérito nacional citado acima, observou-se que cerca de um quarto de todos os entrevistados, incluindo os abstinentes, relataram já terem tido algum problema decorrente do uso de álcool, sendo os mais frequentes os 
problemas físicos $(22 \%)$, familiares $(11 \%)$, sociais (10\%), violência (10\%) e no trabalho (5\%).

Os transtornos relacionados ao uso do álcool representam ainda a oitava causa de concessão de auxílio-doença no sistema previdenciário brasileiro, sendo que os pacientes com problemas relacionados a essa substância utilizam os serviços de saúde até três vezes mais ${ }^{5}$. Outro dado importante é que o uso nocivo de álcool, junto à depressão e às psicoses são as principais causas de transtornos neuropsiquiátricos no Brasil, transtornos que constituem expressiva parcela das doenças crônicas não transmissíveis responsáveis por $72 \%$ das mortes em 20076 .

Entre os diferentes fatores que influenciam o uso de bebidas, estão as expectativas sobre os efeitos do uso de álcool e o resultado que o bebedor espera obter com o consumo. Tais expectativas podem ser aprendidas com a observação de modelos e da experiência direta, sofrendo grande influência do meio e do contexto social, desenvolvendo-se assim desde a infância 7,8. Durante a infância, o comportamento parental e familiar têm grande importância 9. Pais que consomem álcool de forma abusiva e têm atitudes permissivas e expectativas positivas quanto ao consumo tendem a ter filhos dependentes ou que abusam de bebidas, além das consequências negativas que essas crianças sofrem 10,11.

Durante a adolescência, o grupo de amigos torna-se o principal fator de influência sobre as expectativas, e o consumo associa-se ao contexto social, comportamento que se estende ao início da vida adulta. Nesse período, as principais expectativas são redução da tensão, manejo dos problemas, assertividade em situações sociais e melhora da atividade sexual, expectativas positivas que se associam a maior frequência e quantidade de álcool consumido 12 e também ao padrão de beber com embriaguez 13,14.

Há estudos que demonstram que, na adolescência, os homens tendem a apresentar mais expectativas positivas que as mulheres, principalmente quanto à redução da tensão e à celebração com amigos 12,15. Porém, entre adultos jovens, os resultados mostram-se divergentes. Pesquisas entre universitários mostram que não há diferença entre os sexos, e ambos apresentam altos percentuais de expectativas positivas, tais como facilitação das interações sociais, diminuição de emoções negativas, ativação do prazer sexual, efeitos positivos no humor e na avaliação de si mesmo 13. Por outro lado, Corcoran \& Michels 16 encontraram expectativas negativas como tristeza, solidão e situações de risco associadas a situações de ingestão de álcool. Essa diferença aparece ainda em relação à sexualidade; Wilsnack \& Wilsnack 17 mostraram que $60 \%$ das mulheres reportaram desinibição sexual decorrente do uso de álcool, e, apesar de os homens também apresentarem essa experiência, eles tiveram maior associação entre consumo e prazer sexual reportado.

Apesar de o consumo de álcool ter aumentado entre mulheres que apresentam papéis sociais similares aos do homem 18, o uso de bebidas pelo sexo feminino ainda é julgado de forma negativa e mais severa 19 , o que pode ajudar a explicar as diferenças tanto em comportamentos quanto nas expectativas sobre os efeitos do uso de álcool. Nesse sentido, as mulheres mais jovens apresentam mais expectativas positivas quando comparadas às mais velhas 20,21, possivelmente porque o papel feminino está em mudança, com a mulher ocupando, cada vez mais, posições tradicionalmente masculinas 22 .

Compreender as expectativas associadas ao uso de álcool, em especial ao beber se embriagando, pode indicar estratégias de abordagem dos sujeitos, reduzindo a prevalência desse modo de beber. A despeito da importância das expectativas associadas ao uso do álcool, há poucos estudos no país que se debruçaram sobre o tema. Assim, este estudo se propõe a analisar a associação entre beber se embriagando e as expectativas com o uso do álcool, em uma amostra populacional, examinando o papel do gênero nessa associação.

\section{Método}

Este estudo faz parte do Projeto GENACIS (Gender, Alcohol, and Culture: An International Study), uma série de estudos transversais realizados em diferentes países, com objetivo geral de comparar os padrões de uso de álcool entre homens e mulheres em diferentes contextos e culturas, sob a perspectiva de gênero 23 . No presente estudo, foram analisados apenas os dados brasileiros, provenientes da Região Metropolitana de São Paulo. Trata-se, assim, de um estudo transversal, domiciliar, de base populacional, realizado com amostra probabilística estratificada por conglomerados na Região Metropolitana de São Paulo.

\section{Amostragem}

A população deste estudo é constituída por pessoas com 18 anos ou mais de idade, residentes na área urbana dos 39 municípios da Região Metropolitana de São Paulo. O processo de amostragem utilizado foi o da amostragem por conglomerados em dois estágios. Foram sorteados os setores censitários, com probabilidade proporcional ao tamanho do setor, e, posteriormente, 
sorteados os domicílios. Os dados foram analisados para o total da Região Metropolitana de São Paulo. Nesse sentido, o tamanho da amostra foi determinado buscando-se atender às necessidades de precisão das estimativas para a Região Metropolitana de São Paulo, supondo-se uma taxa de não-resposta de $20 \%$, chegando-se, assim, ao número de 2.800 pessoas. Todos os domicílios existentes nos setores censitários sorteados foram previamente arrolados, elaborando-se a listagem de endereços.

\section{Instrumentos}

O questionário GENACIS é composto de 119 questões referentes a variáveis sociodemográficas, avaliação da experiência no trabalho, rede social e variáveis relacionadas ao uso de bebidas (quantidade, frequência e contexto do uso de álcool, consequências do beber). Além de incluir instrumentos para identificação de possíveis casos de dependência, esse questionário detalha o uso por tipo de bebidas, permitindo identificar padrões nocivos do uso de álcool, como o beber com embriaguez, já tendo sido amplamente utilizado no país 18,24.

\section{Expectativas e consequências do uso de álcool}

As expectativas foram investigadas a partir de cinco questões: duas delas sobre expectativas relacionadas à facilitação no relacionamento interpessoal ("quando você bebe, acha mais fácil se abrir com outras pessoas?" e "quando você bebe, acha mais fácil falar com seu atual companheiro sobre seus sentimentos ou problemas?") e três delas sobre expectativas relacionadas à atividade sexual ("quando você bebe, sente-se menos inibido(a) com sexo?", "quando você bebe, acha a atividade sexual mais prazerosa?" e "quando você bebe, sente-se sexualmente mais atraente?”). As perguntas acima foram aplicadas em todos os centros que participaram do estudo GENACIS e foram selecionadas em função de conterem aspectos frequentemente apontados em estudos sobre expectativas relacionadas ao uso de álcool 25,26.

\section{Procedimentos}

Os entrevistadores possuíam experiência prévia com inquéritos domiciliares e foram treinados a partir de role-playings e da aplicação dos questionários, discutindo-se item por item. Para controle de qualidade, $10 \%$ das entrevistas foram checadas em parte dos aspectos investigados. A coleta se deu entre dezembro de 2005 e abril de
2007, sendo, inicialmente, no Município de São Paulo e, posteriormente, nos demais municípios da Região Metropolitana de São Paulo.

\section{Análise dos dados}

Os dados foram digitados em planilha eletrônica, após a qual foram extraídas tabelas de frequência simples, verificada a consistência dos dados e feitas as correções necessárias. A análise dos dados foi feita por meio do módulo survey do programa estatístico Stata 10.0 (Stata Corp., College Station, Estados Unidos), considerando-se o delineamento amostral e os ajustes para as proporções de sexo e faixa etária encontradas na população. Foi feita uma análise descritiva da população estudada com o cálculo de medidas de tendência central e dispersão para variáveis contínuas e de frequência para variáveis categóricas. As estimativas de prevalência e respectivos intervalos de 95\% de confiança foram obtidos aplicando-se pesos que corrigissem para o efeito de amostragem complexa. Na análise univariada, a significância estatística foi avaliada pelo teste de Rao-Scott, por tratar-se de amostra complexa 27.

Para examinar a associação entre beber com embriaguez e as expectativas associadas ao uso de álcool, foram identificados os sujeitos que apresentaram, ao menos, um episódio de embriaguez no ano anterior. Essa foi considerada a variável dependente. A associação da variável dependente com as variáveis explicativas foi investigada por meio das estimativas de odds ratio (OR) simples e ajustadas. Para a análise multivariada, as expectativas foram transformadas em variáveis dicotômicas cujas respostas passaram a ser "não" (nunca teve a expectativa em questão) e "sim" (às vezes e geralmente verdadeiro que tenha tal expectativa). A análise multivariada 28 foi feita pela construção de modelo de regressão logística, com cálculo de OR ajustados. Foram construídos modelos para cada uma das expectativas investigadas, separadas por gênero e ajustando-as para as seguintes variáveis: idade, escolaridade e renda. Tais variáveis foram escolhidas como potenciais confundidores por serem apontadas, pela literatura, como associadas a expectativas 29,30,31,32,33,34,35 e também a padrão de uso de álcool 36,37,38.

\section{Considerações éticas}

Este estudo foi submetido ao Comitê de Ética da Faculdade de Medicina de Botucatu, Universidade Estadual Paulista Júlio de Mesquita Filho - UNESP, tendo sido aprovado em 4 de setembro de 2006 (Ofício nº 437/2006). Só participaram aqueles que voluntariamente o quiseram, me- 
diante a assinatura de termo de consentimento após esclarecimentos, sendo assegurados o sigilo de suas respostas e a possibilidade de desistir a qualquer momento, sem sanções.

\section{Resultados}

Foram entrevistados 2.083 sujeitos, o que corresponde à taxa de resposta de $74,5 \%$. Como o presente estudo investigou as expectativas do uso do álcool entre aqueles que o ingeriram no ano anterior à entrevista, foi incluído apenas esse grupo, o que equivale a 876 pessoas $(44,8 \%$ da amostra inicial).

A Tabela 1 mostra a prevalência de beber com embriaguez segundo as variáveis sociodemográficas. Esse padrão foi encontrado predominantemente entre os homens $(48,4 \%)$, com idades inferiores a 50 anos (variando de $38,3 \%$ a $41,7 \%$ ) e escolaridade até o Ensino Médio incompleto $(42,8 \%)$. Não foi observada associação entre beber com embriaguez e renda per capita $(\mathrm{p}=0,32)$.

A associação entre beber com embriaguez e expectativas do efeito de uso de álcool entre homens é mostrada na Tabela 2. Achar mais fácil se abrir com outras pessoas ( $p<0,001)$, sentir-se menos inibido(a) com sexo ( $\mathrm{p}=0,004)$, achar a atividade sexual mais prazerosa $(\mathrm{p}=0,02)$ e sentirse sexualmente mais atraente $(p=0,02)$ associaram-se a, pelo menos, um episódio de beber com embriaguez no último ano. Já entre as mulheres (Tabela 3), todas as expectativas associaram-se à ocorrência de, ao menos, um episódio de beber com embriaguez no ano anterior $(\mathrm{p}<0,001)$.

A Tabela 4 mostra o modelo de regressão logística para os homens. As expectativas de achar mais fácil se abrir com outras pessoas $(\mathrm{OR}=2,21)$, sentir-se menos inibido(a) com sexo $(\mathrm{OR}=2,43)$,

Prevalência de, ao menos, um episódio de beber com embriaguez no último ano segundo variáveis sociodemográficas, em amostra proveniente da Região Metropolitana de São Paulo *, Brasil $(n=876)$.

\begin{tabular}{|c|c|c|c|c|c|c|}
\hline & \multirow{3}{*}{$\begin{array}{c}\text { Total } \\
\text { n }\end{array}$} & \multicolumn{4}{|c|}{ Episódio de beber com embriaguez } & \multirow[t]{3}{*}{ Valor de $p * \star$} \\
\hline & & \multicolumn{2}{|c|}{ Não } & \multicolumn{2}{|c|}{ Sim } & \\
\hline & & n & $\% * \star \star$ & $\mathbf{n}$ & 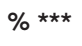 & \\
\hline \multicolumn{7}{|l|}{ Sexo \# } \\
\hline Masculino & 505 & 260 & 51,6 & 245 & 48,4 & $<0,01$ \\
\hline Feminino & 370 & 304 & 81,9 & 66 & 18,1 & \\
\hline \multicolumn{7}{|l|}{ Idade (anos) \#\# } \\
\hline $18-29$ & 300 & 177 & 58,3 & 123 & 41,7 & 0,02 \\
\hline $30-39$ & 219 & 131 & 61,7 & 88 & 38,3 & \\
\hline $40-49$ & 161 & 102 & 59,0 & 59 & 41,0 & \\
\hline $50-59$ & 92 & 72 & 76,6 & 20 & 23,4 & \\
\hline 60 ou mais & 101 & 80 & 78,4 & 21 & 21,6 & \\
\hline \multicolumn{7}{|l|}{ Escolaridade \#\#\# } \\
\hline Não frequentou escola & 431 & 18 & 82,2 & 4 & 17,8 & 0,01 \\
\hline Até o médio incompleto & 421 & 252 & 57,4 & 169 & 42,6 & \\
\hline Médio completo ou mais & 22 & 294 & 66,5 & 137 & 33,6 & \\
\hline \multicolumn{7}{|l|}{ Renda (em Reais) \# } \\
\hline $0,00-150,00$ & 265 & 167 & 64,8 & 98 & 35,2 & 0,32 \\
\hline $151,00-300,00$ & 176 & 120 & 68,4 & 56 & 31,6 & \\
\hline $301,00-600,00$ & 204 & 124 & 58,2 & 80 & 41,8 & \\
\hline 601,00 ou mais & 230 & 153 & 58,5 & 77 & 41,5 & \\
\hline
\end{tabular}

* Foram incluídos apenas os sujeitos que relataram ter bebido no último ano;

** Teste de Rao-Scott 27;

*** Percentuais corrigidos para desenho amostral;

\# Sem informação de 1 sujeito;

\#\# Sem informação de 3 sujeitos;

\#\#\# Sem informação de 2 sujeitos. 
Prevalência de, ao menos, um episódio de beber com embriaguez no último ano e expectativas associadas ao uso de álcool entre homens, em amostra de sujeitos residentes na Região Metropolitana de São Paulo *, Brasil ( $n=508)$.

\begin{tabular}{|c|c|c|c|c|c|}
\hline & \multicolumn{4}{|c|}{ Episódio de beber com embriaguez } & \multirow[t]{3}{*}{ Valor de $p$ ** } \\
\hline & \multicolumn{2}{|c|}{ Não } & \multicolumn{2}{|c|}{ Sim } & \\
\hline & $\mathrm{n}$ & $\% * \star *$ & $\mathrm{n}$ & $\%$ *** & \\
\hline \multicolumn{6}{|c|}{ Acha mais fácil se abrir com outras pessoas } \\
\hline Nunca & 184 & 59,5 & 125 & 40,5 & \multirow[t]{3}{*}{$<0,001$} \\
\hline Às vezes, verdadeiro & 37 & 33,0 & 58 & 67,0 & \\
\hline Geralmente, verdadeiro & 39 & 44,7 & 65 & 55,3 & \\
\hline \multicolumn{6}{|c|}{ Acha mais fácil falar com seu atual companheiro sobre seus sentimentos ou problemas? \# } \\
\hline Nunca & 171 & 54,3 & 151 & 45,7 & \multirow{3}{*}{0,22} \\
\hline Às vezes, verdadeiro & 22 & 43,0 & 27 & 57,0 & \\
\hline Geralmente, verdadeiro & 25 & 47,3 & 28 & 52,7 & \\
\hline \multicolumn{6}{|c|}{ Sente-se menos inibido(a) com sexo?\#\# } \\
\hline Nunca & 219 & 56,3 & 174 & 43,7 & \multirow[b]{3}{*}{0,004} \\
\hline Às vezes, verdadeiro & 20 & 28,2 & 33 & 71,8 & \\
\hline Geralmente, verdadeiro & 20 & 42,3 & 40 & 57,7 & \\
\hline \multicolumn{6}{|c|}{ Acha a atividade sexual mais prazerosa? \#\#\# } \\
\hline Nunca & 220 & 55,4 & 181 & 44,6 & \multirow[b]{3}{*}{0,02} \\
\hline Às vezes, verdadeiro & 18 & 32,1 & 32 & 67,9 & \\
\hline Geralmente, verdadeiro & 21 & 39,4 & 35 & 60,6 & \\
\hline \multicolumn{6}{|c|}{ Sente-se sexualmente mais atraente? \#\#\# } \\
\hline Nunca & 228 & 55,2 & 188 & 44,8 & \multirow{3}{*}{0,02} \\
\hline Às vezes, verdadeiro & 15 & 29,2 & 30 & 70,8 & \\
\hline Geralmente, verdadeiro & 17 & 40,0 & 30 & 60,0 & \\
\hline
\end{tabular}

* Foram incluídos apenas os sujeitos que relataram ter bebido no último ano.

** Teste de Rao-Scott 27;

*** Percentuais corrigidos para desenho amostral;

\# Apenas entre aqueles que têm companheiro;

\#\# Sem informação de 2 sujeitos;

\#\#\# Sem informação de 3 sujeitos.

achar a atividade sexual mais prazerosa $(\mathrm{OR}=$ $2,21)$ e sentir-se sexualmente mais atraente $(\mathrm{OR}=$ 2,38 ) mostraram-se associadas ao maior risco de apresentar episódio de beber com embriaguez, mesmo ajustando-se o modelo para idade, escolaridade e renda per capita.

Resultado similar foi encontrado entre as mulheres, como demonstra a Tabela 5. Entre elas, associaram-se a maior risco de episódio de beber com embriaguez as expectativas de achar mais fácil se abrir com outras pessoas (OR $=3,39$ ), sentir-se menos inibido(a) com sexo $(\mathrm{OR}=3,34)$, achar a atividade sexual mais prazerosa $(\mathrm{OR}=$ $3,12)$ e sentir-se sexualmente mais atraente $(\mathrm{OR}=$ 2,68). A expectativa de achar mais fácil falar com companheiro não se mostrou associado ao padrão investigado para ambos os sexos.

\section{Discussão}

Neste estudo, observou-se que, tanto para homens quanto para mulheres, as expectativas de achar mais fácil se abrir com outras pessoas, sentir-se menos inibido(a) em relação ao sexo, achar a atividade sexual mais prazerosa e sentirse sexualmente mais atraente mantiveram-se independentemente associadas a maior risco de beber com embriaguez mesmo após ajuste para renda, idade e escolaridade. A expectativa achar mais fácil falar com seu atual companheiro sobre seus sentimentos ou problemas não se associou a beber com embriaguez em nenhum dos gêneros analisados.

Neste levantamento, a maioria das pessoas relatou que nunca teve qualquer das expectativas investigadas em relação à ingestão de bebidas, o 
Ocorrência de, ao menos, um episódio de beber com embriaguez no último ano e expectativas associadas ao uso de álcool entre mulheres, em amostra de sujeitos residentes na Região Metropolitana de São Paulo *, Brasil $(n=370)$.

\begin{tabular}{|c|c|c|c|c|c|}
\hline & \multicolumn{4}{|c|}{ Episódio de beber com embriaguez } & \multirow[t]{3}{*}{ Valor de $p$ ** } \\
\hline & \multicolumn{2}{|c|}{ Não } & \multicolumn{2}{|c|}{ Sim } & \\
\hline & $\mathrm{n}$ & $\% * \star \star$ & $\mathbf{n}$ & $\% * \star \star$ & \\
\hline \multicolumn{6}{|c|}{ Acha mais fácil se abrir com outras pessoas? \# } \\
\hline Nunca & 227 & 87,7 & 36 & 12,3 & $<0,001$ \\
\hline Às vezes, verdadeiro & 47 & 77,6 & 13 & 22,4 & \\
\hline Geralmente, verdadeiro & 28 & 55,9 & 17 & 44,1 & \\
\hline \multirow{2}{*}{\multicolumn{6}{|c|}{$\begin{array}{l}\text { Acha mais fácil falar com seu atual companheiro sobre seus sentimentos ou } \\
\text { problemas? \#\# }\end{array}$}} \\
\hline & & & & & \\
\hline Nunca & 200 & 85,7 & 39 & 14,3 & $<0,001$ \\
\hline Às vezes, verdadeiro & 21 & 91,8 & 1 & 8,2 & \\
\hline Geralmente, verdadeiro & 15 & 50,4 & 9 & 49,6 & \\
\hline \multicolumn{6}{|c|}{ Sente-se menos inibido(a) com sexo? \#\#\# } \\
\hline Nunca & 245 & 86,8 & 40 & 13,2 & $<0,001$ \\
\hline Às vezes, verdadeiro & 27 & 77,6 & 9 & 22,4 & \\
\hline Geralmente, verdadeiro & 27 & 53,8 & 17 & 46,2 & \\
\hline \multicolumn{6}{|c|}{ Acha a atividade sexual mais prazerosa? § } \\
\hline Nunca & 248 & 86,1 & 42 & 13,9 & $<0,001$ \\
\hline Às vezes, verdadeiro & 22 & 86,8 & 6 & 13,2 & \\
\hline Geralmente, verdadeiro & 27 & 50,9 & 18 & 49,1 & \\
\hline \multicolumn{6}{|c|}{ Sente-se sexualmente mais atraente? §§ } \\
\hline Nunca & 247 & 86,2 & 41 & 13,8 & $<0,001$ \\
\hline Às vezes, verdadeiro & 33 & 86,0 & 7 & 14,0 & \\
\hline Geralmente, verdadeiro & 21 & 49,8 & 18 & 50,2 & \\
\hline
\end{tabular}

* Foram incluídos apenas os sujeitos que relataram ter bebido no último ano;

** Teste de Rao-Scott 27;

*** Percentuais corrigidos para desenho amostral;

\# Sem informação de 2 sujeitos:

\#\# Apenas entre aqueles que têm companheiro;

\#\#\# Sem informação de 5 sujeitos;

§ Sem informação de 7 sujeitos;

$\S \S$ Sem informação de 3 sujeitos.

que contraria Monahan \& Lannutti 39, segundo os quais o álcool, considerado pela concepção popular como um elemento responsável por acalmar a ansiedade e o nervosismo, ajudaria a promover a interação entre pares e com o sexo oposto, funcionando como facilitador do contato social e proporcionando maior prazer sexual. No entanto, essa baixa taxa de resposta afirmativa foi encontrada também em outros países como o Uruguai, o que pode sugerir semelhança entre países sul-americanos, como também receio de julgamentos morais quanto às expectativas relativas ao comportamento de beber.

Entre os entrevistados, tanto homens quanto mulheres, apresentaram expectativas positivas sobre o consumo de álcool. A única exceção foi a expectativa "achar mais fácil falar com seu atual companheiro sobre seus sentimentos ou problemas". Essa expectativa, embora apresentasse associação na análise univariada, não se manteve associada após os ajustes na regressão logística. Esse achado destoa do observado por Caudill et al. 40, que demonstraram que homens que ingeriram bebidas alcoólicas e acreditavam que suas parceiras também o haviam feito apresentaram tendência em falar de si e com maior nível de intimidade. Do mesmo modo, estudo de Borjesson \&Dunn 41 demonstrou que tanto homens quanto mulheres apresentam a expectativa de melhora de interação social com o álcool. Demonstrando 
Tabela 4

Modelo de regressão logística para ocorrência de beber com embriaguez no último ano entre homens residentes na Região Metropolitana de São Paulo, Brasil ( $n=508)$.

\begin{tabular}{|c|c|c|c|c|c|}
\hline Homens & OR simples & IC95\% & OR ajustado * & $\mathrm{IC} 95 \%$ & Valor de $\mathrm{p}$ \\
\hline \multicolumn{6}{|c|}{ Acha mais fácil se abrir com outras pessoas } \\
\hline Sim & 1,00 & & & & \\
\hline Não & 2,32 & $1,46-3,69$ & 2,21 & $1,39-3,53$ & 0,001 \\
\hline \multicolumn{6}{|c|}{ Acha mais fácil falar com seu atual companheiro sobre seus sentimentos } \\
\hline \multicolumn{6}{|c|}{ ou problemas? } \\
\hline Não & 1,00 & & & & 0,13 \\
\hline Sim & 1,46 & $0,89-2,40$ & 1,48 & $0,89-2,47$ & \\
\hline \multicolumn{6}{|c|}{ Sente-se menos inibido(a) com sexo? } \\
\hline Não & 1,00 & & & & 0,003 \\
\hline Sim & 2,39 & $1,34-4,24$ & 2,43 & $1,37-4,32$ & \\
\hline \multicolumn{6}{|c|}{ Acha a atividade sexual mais prazerosa? } \\
\hline Não & 1,00 & & & & 0,02 \\
\hline Sim & 2,25 & $1,20-4,21$ & 2,21 & $1,16-4,19$ & \\
\hline \multicolumn{6}{|c|}{ Sente-se sexualmente mais atraente? } \\
\hline Não & 1,00 & & & & 0,01 \\
\hline Sim & 2,36 & $1,25-4,46$ & 2,38 & $1,24-4,59$ & \\
\hline
\end{tabular}

IC95\%: intervalo de 95\% de confiança; OR: odds ratio.

* Cada uma das expectativas foi ajustada para idade, escolaridade e renda per capita.

Tabela 5

Modelo de regressão logística para ocorrência de beber com embriaguez no último ano entre mulheres residentes na Região Metropolitana de São Paulo, Brasil $(n=370)$.

\begin{tabular}{|c|c|c|c|c|c|}
\hline Mulheres & OR simples & IC95\% & OR ajustado * & IC95\% & Valor de p \\
\hline \multicolumn{6}{|c|}{ Acha mais fácil se abrir com outras pessoas } \\
\hline $\operatorname{Sim}$ & 1,00 & & & & \\
\hline Não & 3,52 & $1,74-7,09$ & 3,39 & $1,62-7,08$ & 0,002 \\
\hline \multicolumn{6}{|c|}{ Acha mais fácil falar com seu atual companheiro sobre seus } \\
\hline \multicolumn{6}{|c|}{ sentimentos ou problemas? } \\
\hline Não & 1,00 & & & & \\
\hline $\operatorname{Sim}$ & 2,68 & $1,18-6,09$ & 2,36 & $0,94-5,91$ & 0,07 \\
\hline \multicolumn{6}{|c|}{ Sente-se menos inibido(a) com sexo? } \\
\hline Não & 1,00 & & & & \\
\hline $\operatorname{Sim}$ & 3,67 & $1,90-7,09$ & 3,34 & $1,72-6,47$ & 0,001 \\
\hline \multicolumn{6}{|c|}{ Acha a atividade sexual mais prazerosa? } \\
\hline Não & 1,00 & & & & \\
\hline $\operatorname{Sim}$ & 3,50 & $1,81-6,77$ & 3,12 & $1,53-6,40$ & 0,002 \\
\hline \multicolumn{6}{|c|}{ Sente-se sexualmente mais atraente? } \\
\hline Não & 1,00 & & & & \\
\hline Sim & 3,04 & $1,81-5,10$ & 2,68 & $1,55-4,64$ & 0,001 \\
\hline
\end{tabular}

IC95\%: intervalo de 95\% de confiança; OR: odds ratio.

* Cada uma das expectativas foi ajustada para idade, escolaridade e renda per capita. 
a falta de consenso, um estudo de 199742 encontrou que o álcool inibiu as interações sociais em homens e mulheres quanto a falarem de si. É preciso destacar que os estudos citados utilizaram instrumentos diferentes, o que pode justificar os achados distintos.

Os resultados que mostram maior facilidade de o homem falar sobre questões pessoais sob efeito do álcool podem sugerir que, neles, a bebida diminua a ansiedade e permita falar mais sobre aspectos íntimos, o que não é culturalmente aceito ou estimulado em situações cotidianas. Já para as mulheres, o aumento no nível de intimidade das interações sociais pode ser condenado, uma vez que implicaria em perda de controle e até situações que as exponham a algum tipo de risco. No presente estudo, homens e mulheres mostraram associação entre embriaguez e expectativas positivas quanto à sexualidade. De fato, alguns trabalhos mostram que mulheres podem apresentar expectativas positivas em relação ao uso de álcool, mas também podem apresentar medo e ansiedade quanto às possíveis consequências negativas, como assédio e violência sexual 43 .

Nesse sentido, as expectativas relacionadas à sexualidade também mostram resultados controversos. Estudos conduzidos na República Tcheca, Espanha, Nigéria, Uganda, Sri Lanka, Costa Rica e Uruguai não observaram diferenças entre homens e mulheres 26. Já na Índia, Japão e Uganda, os homens apresentaram mais expectativas positivas em relação à sexualidade; enquanto a Suécia foi o único país onde as mulheres apresentaram maior taxa de expectativas em relação à melhora da atividade sexual do que os homens 26 .

De grande importância para a área da saúde é o achado que, tanto entre os homens como entre as mulheres, as expectativas positivas em relação ao uso do álcool se associaram a beber com embriaguez, independentemente dos critérios de idade, renda e escolaridade. Particularmente, em relação à idade, esse achado coincide com estudo de Leigh \& Stacy 44 , segundo os quais há muitos estudos sobre uso de bebidas entre estudantes universitários, mostrando mais expectativas positivas nesse grupo quando comparado a outros grupos etários. Esse dado sugere que esses estudantes ainda não apresentaram consequências negativas decorrentes do uso de bebidas, pois os autores ressaltam que, em todas as faixas etárias, as expectativas positivas se associam a uso de álcool, o que sugere que a idade pode estar associada ao uso per se.

Sobreira 45 , analisando as expectativas com uso de álcool entre agentes comunitários de saúde, observou que os homens tinham expectativas mais altas que as mulheres e, ao se compararem diferentes faixas de idade, os mais jovens tinham expectativas mais elevadas que os mais velhos. No presente estudo, a inclusão de idade na análise multivariada permitiu visualizar a associação de cada uma das expectativas com o beber com embriaguez, controlando para essa variável. Em outras palavras, expectativas positivas se mantiveram associadas a beber com embriaguez independentemente da idade.

No entanto, as expectativas formadas anteriormente à idade adulta parecem ter importância fundamental em comportamentos futuros. Estudo longitudinal 29 encontrou que expectativas positivas na adolescência foram fortes preditores de uso nocivo de álcool quando os sujeitos foram reavaliados cerca de 20 anos após, o que sugere que as expectativas positivas, se encontradas em jovens, tendem a permanecer em idades posteriores. Além disso, as expectativas negativas não foram importantes em influenciar o padrão de ingestão do álcool; o que sugere que programas de prevenção deveriam focar em estratégias de redução de danos, promovendo a ideia de que é melhor usar o álcool com moderação, defendendo que essa abordagem seria mais eficiente do que aquelas focadas nas consequências negativas 29 .

Como dito anteriormente, as expectativas em relação ao uso de álcool são construídas historicamente na vida do sujeito a partir de modelos de comportamentos parentais e de amigos, pelas experiências diretas e pela influência da mídia, entre outros 29,46. Em um estudo longitudinal com adolescentes 47 , observou-se que a exposição a diferentes formas de propaganda de bebidas alcoólicas foi preditora do uso de bebidas no futuro, embora haja influência das experiências anteriores dos sujeitos com o álcool. Uma pesquisa realizada com estudantes brasileiros 48 também encontrou associação entre apreciação e exposição a propagandas de bebidas alcoólicas e o consumo pelos adolescentes.

Em artigo de revisão, Pinsky \& El Jundi 49 observaram que fatores como exposição à publicidade e a atratividade de bebidas alcoólicas estão relacionadas a maiores expectativas de consumo e também a uso de maiores quantidades e mais precocemente, resultado também encontrado em trabalho de Snyder et al. 50. Outro dado alarmante é que, em contrapartida, o conteúdo das campanhas de prevenção sobre uso de álcool e outras drogas veiculadas pela mídia raramente era lembrado pelos adolescentes.

De acordo com os dados da literatura, o padrão de beber com embriaguez está associado principalmente à menor idade (adultos jovens), os quais apresentam mais expectativas positivas em relação às consequências do uso de álcool. 
Desse modo, é preciso criar políticas que atinjam essa população e que tenham como objetivo alterar essas expectativas, o que pode influenciar diretamente no consumo. Uma das medidas que poderia ser tomada é a mudança ou restrição aos anúncios de bebidas, que, atualmente, associam consequências positivas ao ato de beber, como sensações de bem-estar e facilitação de interações sociais. Recentemente, passaram a ser veiculadas propagandas educativas com as consequências ruins do uso nocivo de bebidas, no entanto, são medidas pontuais, sem continuidade e podem ser menos eficientes, como demonstra o estudo de Patrick et al. 29.

De acordo com um levantamento da Organização Mundial da Saúde 51, o Brasil tem falhado em implementar políticas públicas de controle sobre o uso do álcool, sendo que o acesso é facilitado, inclusive, entre menores de idade ${ }^{52}$. O Brasil ainda apresenta estratégias insuficientes relacionadas ao controle do álcool, com preços relativamente baixos, sem obrigatoriedade de controle sobre os motoristas e com propagandas que veiculam experiências altamente positivas do uso do álcool 51.

Este estudo deve ser avaliado à luz de suas limitações: tratando-se de estudo transversal, não se pode atribuir direção de causalidade entre expectativas e uso de álcool com embriaguez. As expectativas foram examinadas a partir de questões extraídas de instrumentos padronizados e não a partir da utilização desses instrumentos, o que dificulta a comparação com outras pesquisas que não tenham feito parte do estudo GENACIS. Embora a taxa de resposta tenha sido um pouco menor que a esperada, foi ainda maior que a de estudos similares conduzidos recentemente no país, no que diz respeito à coleta nas grandes cidades 2 .

As expectativas são comportamentos aprendidos por experiências diretas e observação de modelos, estimulados ainda pela cultura e pelo significado social do beber em nossa sociedade. No entanto, há poucas pesquisas que investigam, no Brasil, essas expectativas e sua relação com o uso de álcool em amostras populacionais e que seriam fundamentais para subsidiar estratégias de intervenção e prevenção. Por fim, são necessárias políticas públicas que enfoquem a mudança dessas expectativas, bem como a difusão de modelos mais saudáveis do uso do álcool pela sociedade.

\section{Resumo}

O objetivo deste trabalho foi investigar as expectativas de homens e mulheres com relação ao uso do álcool e a associação dessas com o comportamento de beber com embriaguez. Foi realizado inquérito epidemiológico, domiciliar, transversal, de base populacional, com amostra probabilística estratificada por conglomerados, na Região Metropolitana de São Paulo, Brasil. Foram entrevistadas 2.083 pessoas de ambos os sexos utilizando-se o questionário GENACIS (Gender, Alcohol and Culture: An International Study). Beber com embriaguez foi considerada variável dependente, e foram construídos modelos de regressão logística para cada sexo, ajustando-se os modelos para idade, escolaridade e renda. Todas as expectativas, exceto achar mais fácil falar com companheiro, associaram-se ao comportamento de beber com embriaguez. Nosso estudo mostrou que beber com embriaguez pode estar associado a expectativas com uso do álcool. Compreender essas expectativas pode contribuir para elaboração de estratégias mais efetivas de prevenção do beber excessivo.

Consumo de Bebidas Alcoólicas; Abuso de Álcool; Comportamento 


\section{Colaboradores}

M. C. P. Lima participou na elaboração do estudo, na análise estatística, na interpretação dos dados e na redação final do manuscrito. M. B. Cavariani, J. B. Oliveira e F. Kerr-Corrêa participaram na elaboração do estudo, na interpretação dos dados e na redação final do manuscrito.

\section{Agradecimentos}

Este estudo fez parte do Projeto GENACIS e contou com o financiamento FAPESP no. 04/11729-2. M. B. Cavariani foi bolsista CAPES.

\section{Referências}

1. World Health Organization. The World Health report 2004 - global status report on alcohol. Geneva: World Health Organization; 2004.

2. Laranjeira R, Pinsky I, Sanches M, Zaleski M, Caetano R. Alcohol use patterns among Brazilian adults. Rev Bras Psiquiatr 2010; 32:231-41.

3. National Institute of Alcohol Abuse and Alcoholism. Underage drinking: a major public health challenge. Alcohol Alert 2003; 59:1-4.

4. Oliveira JB, Lima MCP, Simão MO, Cavariani MB, Tucci AM, Kerr-Corrêa F. Violência entre parceiros íntimos e álcool: prevalência e fatores associados. Rev Panam Salud Pública 2009; 26:494-501.

5. Odo AS, Araújo AC, Santos AF, Toledo FCP, Yonamine M, Silva AO, et al. Indicações e limites das análises toxicológicas para substâncias psicoativas. Rev Psiquiatr Clin (São Paulo) 2000; 7:50-6.

6. Schmidt MI, Duncan BB, Azevedo-Silva G, Menezes AM, Monteiro CA, Barreto SM, et al. Chronic non-communicable diseases in Brazil: burden and current challenges. Lancet 2011; 377:1949-61.

7. Bandura A. Social learning theory. New York: General Learning Press; 1977.

8. Bandura A. Social foundations of thought and action. Englewood Cliffs: Prentice-Hall; 1986.

9. Clark DB. The natural history of adolescent alcohol use disorders. Addiction 2004; 99:5-22.

10. Clark DB, Lesnick L, Hegedus AM. Traumas and other adverse life events in adolescents with alcohol abuse and dependence. J Am Acad Child Adolesc Psychiatry 1997; 36:1744-51.

11. Barnow S, Schckit MA, Lucht M, John U, Freyberger HJ. The importance of a positive family history of alcoholism, parental rejection and emotional warmth, behavioral problems and peer substance use for alcohol problems in teenagers: a path analysis. J Stud Alcohol 2002; 63:305-15.
12. Wiers RW, Hoogeveen KJ, Sergeant JA, Gunning WB. High and low dose alcohol related expectancies and the differential associations with drinking in male and female adolescents and young adults. Addiction 1997; 92:871-88.

13. Peuker AC, Fogaça J, Bizarro L. Expectativas e beber problemático entre universitários. Psicol Teor Pesqui 2006; 22:193-200.

14. Miller P, Plant M, Plant M. Spreading out or concentrating weekly consumption: alcohol problems and other consequences within a UK population sample. Alcohol Alcohol 2005; 40:461-8.

15. Kairouz S, Gliksman L, Demers A, Adalf E. For all these reasons, I do...drink: a multilevel analysis of contextual reasons for drinking among Canadian undergraduates. J Stud Alcohol 2002; 63:600-8.

16. Corcoran KJ, Michels JL. A prototype analysis of psychological situations through the lens of alcohol expectancies and gender. Addict Behav 1998; 23:685-91.

17. Wilsnack SC, Wilsnack R. Drinking and problem drinking in US women. In: Galanter M, editor. Recent developments in alcoholism. New York: Plenum Press; 1995. p. 29-60.

18. Kerr-Correa F, Tucci AM, Hegedus AM, Trinca LA, Oliveira JB, Floripes TMF, et al. Diferenças nos padrões de consumo de álcool entre homens e mulheres em duas comunidades brasileiras distintas. Rev Bras Psiquiatr 2008; 30:235-42.

19. Wilsnack S, Wilsanck R. Epidemiology of women's drink. J Subst Abuse 1991; 3:133-57.

20. Satre DD, Knight BG. Alcohol expectancies and their relationship to alcohol use: age and sex differences. Aging Ment Health 2001; 5:73-83.

21. Lundahl LH, Davis TM, Adesso VJ. Alcohol expectancies: effects of gender, age, and family history of alcoholism. Addict Behav 1997; 22:115-25. 
22. Wilsnack R, Wilsnack SC, Obot IS. Why study gender, alcohol and culture? In: Obot IS, Room R, editores. Alcohol, gender and drinking problems: perspectives from low and middle income countries. Geneva: World Health Organization; 2005. p. 1-23.

23. Taylor B, Rehm J, Aburto JTC, Bejarano J, Cayetano C, Kerr-Corrêa, F, et al. Alcohol, gender, culture and harms in the Americas: PAHO multicentric study final report. Washington DC: Pan American Health Organization; 2007.

24. Laranjeira, R, Pinsky I, Zaleski M, Caetano R. I Levantamento nacional sobre os padrões de consumo de álcool na população brasileira. Brasília: Secretaria Nacional Antidrogas; 2007.

25. Wilsnack SC, Plaud JJ, Wilsnack RW, Klassen AD. Sexuality, gender, and alcohol use. In: Wilsnack RW, Wilsnack SC, editors. Gender and alcohol: individual and social perspectives. New Brunswick: Rutgers Center of Alcohol Studies; 1997. p. 250-88.

26. Bolgren A, Kristjanson A, Wilsnack S. The relationship between sexuality-related alcohol expectancies and drinking across cultures. African Journal of Drug and Alcohol Studies 2007; 1:1-16.

27. Lee ES, Forthofer RN. Analyzing complex survey data. $2^{\text {nd }}$ Ed. Beverly Hills: Sage; 2006.

28. Hosmer DW, Lemeshow S. Applied logistic regression. 2nd Ed. New York: John Wiley \& Sons Inc.; 1989.

29. Patrick ME, Wray-Lake L, Finlay AK, Maggs JL. The long arm of expectancies: adolescent alcohol expectancies predict adult alcohol use. Alcohol Alcohol 2010; 45:17-24.

30. Leigh BC, Stacy AW. Alcohol expectancies and drinking in different groups. Addiction 2004; 99:215-27.

31. Brown SA, Goldman MS, Christiansen BA. Do alcohol expectancies mediate drinking patterns of adults? J Consult Clin Psychol 1985; 53:512-9.

32. Pastor AD, Evans SM. Alcohol outcome expectancies and risk alcohol use problems in women with and without a family history of alcoholism. Drug Alcohol Depend 2003: 70:201-14.

33. Auld MC. Smoking, drinking and income. J Hum Resour 2005; 40:505-18.

34. Obot IS, Room R. Alcohol, gender and drinking problems: perspectives from low and middle income countries. Geneva: World Health Organization; 2005.

35. Elgar FJ, Roberts C, Parry-Longdon N, Boyce W. Income inequality and alcohol use: a multilevel analysis of drinking and drunkenness in adolescents in 34 countries. Eur J Public Health 2005; 15:245-50.

36. Hawkins JD, Graham JW, Maguin E, Abbott R, Hill KG, Catalano RF. Exploring the effects of age of alcohol use initiation and psychosocial risk factors on subsequent alcohol misuse. J Stud Alcohol 1997; 58:280-90.

37. DeWit DJ, Adlaf EM, Offord DR, Ogborne AC. Age at first alcohol use: a risk factor for the development of alcohol disorders. Am J Psychiatry 2000; 157:745-50.
38. Grant BF. The impact of a family history of alcoholism on the relationship between age at onset of alcohol use and DSM-IV alcohol dependence. Alcohol Health Res World 1998; 22:144-7.

39. Monahan JL, Lannutti PJ. Alcohol as social lubricant. Alcohol myopia theory, social self-esteem, and social interaction. Human Communication Research 2000; 26:175-202.

40. Caudill BD, Wilson GT, Abrams DB. Alcohol and self-disclosure: analyses of interpersonal behavior in male and female social drinkers. J Stud Alcohol 1987; 48:401-9.

41. Borjesson WI, Dunn ME. Alcohol expectancies of women and men in relation to alcohol use and perceptions of the effects of alcohol on the opposite sex. Addict Behav 2001; 26:707-19.

42. Schippers GM, De Boer M, van der Staak CPF. Effects of alcohol and expectancy on self-disclosure and anxiety in male and female social drinkers. Addict Behav 1997; 22:305-14.

43. Leigh BC, Aramburu B. The role of alcohol and gender in choices and judgments about hypothetical sexual encounters. Journal of Applied Social Psychology 1996; 26:20-30.

44. Leigh BC, Stacy AW. Alcohol expectancies and drinking in different groups. Addiction 2004; 99:215-27.

45. Sobreira EST. Agentes comunitários de saúde: expectativas e crenças acerca do uso de álcool [Dissertação de Mestrado]. Ribeirão Preto: Faculdade de Medicina de Ribeirão Preto, Universidade de São Paulo; 2009.

46. Wall AM, Thrussell C, Lalonde R. Do alcohol expectancies become intoxicated outcomes? A test of social-learning theory in a naturalistic bar setting. Addict Behav 2003; 28:1271-83.

47. Ellickson PL, Collins RL, Hambarsoomians K, McCaffrey DF. Does alcohol advertising promote adolescent drinking? Results from a longitudinal assessment. Addiction 2005; 100:235-46.

48. Vendrame A, Pinsky I, Faria R, Silva R. Apreciação de propagandas de cerveja por adolescentes: relações com a exposição prévia às mesmas e o consumo de álcool. Cad Saúde Pública 2009; 25:359-65.

49. Pinsky I, El Jundi SARJ. O impacto da publicidade de bebidas alcoólicas sobre o consumo entre jovens: revisão da literatura internacional. Rev Bras Psiquiatr 2008; 30:362-74.

50. Snyder LB, Milici FF, Slater M, Sun H, Strizhakova Y. Effects of alcohol advertising exposure on drinking among youth. Arch Pediatr Adolesc Med 2006; 160:18-24.

51. Babor TF, Caetano R. Evidence-based alcohol policy in the Americas: strengths, weaknesses, and future challenges. Rev Panam Salud Pública 2005; 18:327-37.

52. Romano M, Duailibi S, Pinsky I, Laranjeira R. Pesquisa de compra de bebidas alcoólicas por adolescentes em duas cidades do Estado de São Paulo - SP. Rev Saúde Pública 2007; 41:495-501.

Recebido em 30/Out/2011

Versão final reapresentada em 01/Abr/2012 Aprovado em 03/Abr/2012 